\title{
Minimum error image reconstruction for coded mask telescopes
}

\author{
R.M. Rideout and G.K. Skinner \\ School of Physics and Space Research, The University of Birmingham, Birmingham, B15 2TT, UK \\ Received March 11; accepted May 2, 1996
}

\begin{abstract}
A new method is proposed for reconstructing the data from coded aperture $\mathrm{X} / \gamma$-ray telescopes. This technique, based on the Wiener criterion, is essentially a generalisation of existing Wiener filtering methods but is not confined to systems with position independent point source response functions. The reconstruction method trades off the image degrading effects of statistical detector noise with the effects of imperfect source field coding, to produce a minimum error source field estimate. It offers advantages if the coding properties of the mask-detector configuration considered are not 'ideal' and is particularly useful where multiple dithered observations are made of a given source field. The proposed method is applied to simulated data and to data from a recent balloon flight of an experimental coded mask telescope. It is shown to give better quality images than those formed by more conventional methods.
\end{abstract}

Key words: X-rays: general — techniques: image processing — gamma-rays: observations — telescopes

\section{Introduction}

Coded aperture techniques (for reviews see, e.g. Caroli et al. 1987; Skinner \& Ponman 1995) have been widely investigated and implemented in a variety of telescopes to image astronomical sources of $\mathrm{X} / \gamma$-ray energies above $\sim 10 \mathrm{keV}$ where the effectiveness of grazing incidence optics breaks down.

Coded aperture imaging is a two-stage process whereby the source field information is first coded by modulating the source flux with a coded mask and then decoded using a suitable reconstruction technique. Masks such as those proposed by Gunson \& Polychronopulos (1976) and Fenimore \& Cannon (1978) are commonly used which have certain 'ideal' coding properties and are generally referred to as Uniformly Redundant Arrays (URAs). Unlike random patterns (Dicke 1968), the URAs possess a modulation transfer function similar to that of a finite pinhole (Fenimore 1980) and are characterized by a deltafunction (discrete) cyclic autocorrelation function. In the common cases where image reconstruction is by crosscorrelating the detector data with some mathematical representation of the mask pattern (e.g. Fenimore 1978; Fenimore \& Cannon 1978), the point source response function (PSF) is then also a delta-function. The resulting image is free from 'ghost' peaks, hence fluctuations due to imperfect coding (coding noise) are eliminated.

Since the introduction of URAs other patterns have been proposed, such as the geometric coded masks (Gourlay \& Stephen 1983) and the Modified URAs

Send offprint requests to: G.K. Skinner
(Gottesman \& Fenimore 1989), which possess deltafunction PSFs when cross-correlation is with a pattern slightly different from the original coded mask. With the exception of the MURAs, however, most of these patterns have low transmission (Caroli et al. 1987) and are therefore not likely to be well suited to astronomical applications (Rideout 1996).

It is not always possible to design a telescope capable of utilising the perfect coding properties of an ideal mask pattern. Sometimes the size and shape of the aperture are dictated by instrumental requirements or by other constraints and masks with imperfect coding properties must be used. On other occasions accepting imperfect coding allows the losses associated with a collimator to be avoided (Sims et al. 1980).

\section{Linear reconstruction methods}

The coding of the source flux passing through a coded aperture onto a position sensitive detector can be expressed in a matrix formulation by the 'coding equation',

$$
[D]=[M][S]+[B]+[N]
$$

where $[D]$ represents the binned counts recorded at the detector from the source field distribution $[S]$. The matrix $[M]$ is the coding matrix where $m_{1 i}, \ldots, m_{p i}$ represent the mask transmissions in the line of sight between detector elements $d_{1}, \ldots, d_{p}$ and the $i$-th source element, respectively. Background detector events not modulated by the coded mask are described by the matrix $[B]$, and $[N]$ represents 
the fluctuation in the detector rates due to counting statistics. We here consider cases where the detector counts are dominated by the background rate such that the statistical noise is independent of the source flux.

In expanded form Eq. (1) is

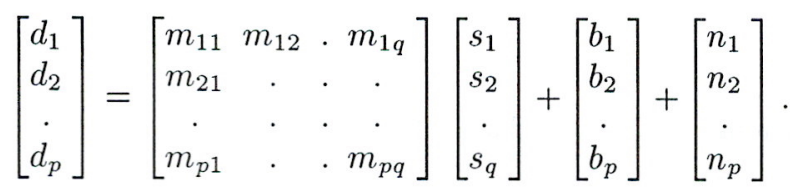

In this form it is easily seen how it is possible to include temporally coded information by extending the number of rows of the matrices (with the exception of $[S]$ ) from $p$, to $t p$, where there are $t$ time bins. This caters for the situation where either the form of the mask is changed as a function of time or where multiple telescope pointing directions occur, either as a result of drift or as part of a 'dithering' strategy to improve the imaging performance.

Linear methods of image reconstruction are all equivalent to pre-multiplying the detector array $[D]$ with a suitable reconstruction matrix $[G]$, forming an estimate $[\hat{S}]$ of the original source field distribution. Reconstruction can then be expressed by the 'decoding equation',

$$
\begin{aligned}
{[\hat{S}] } & =[G][D] \\
& =[G][M][S]+[G][B]+[G][N] .
\end{aligned}
$$

The estimate of a particular source pixel $\hat{s}_{i}$ is given by

$$
\hat{s}_{i}=\sum_{j} g_{i j} d_{j}
$$

and is subject to an uncertainty $\sigma_{\hat{s}_{i}}$. This uncertainty consists of two components, which we will term 'random noise' and 'coding noise'.

Random noise is simply the result of statistical detector noise, $[N]$. Coding noise arises when, for a particular reconstruction matrix, $[G][M]$ is not equal to the identity matrix, $[I]$. If $[M]$ is square and non-singular, it is possible to eliminate coding noise by selecting a reconstruction matrix that is the inverse of $[M]$. Unfortunately, most mask-detector configurations are such that $[M]^{-1}$ contains large terms which amplify the statistical noise and hence the random noise component degrades the reconstructed image beyond practicality.

In cases where a uniform detector background dominates the observed counts, $\mathbf{E}\left(n_{j}^{2}\right)$ (where $\mathbf{E}$ is the expectation operator) is constant for all $j$, equal to $\sigma_{d}^{2}$, say. The average value of the variance due to random noise on each source estimate in a $q$ element source field is then given by (e.g., Caroli et al. 1984; Sembay \& Gehrels 1990),

$$
\begin{aligned}
\sigma_{\hat{s}}^{2} & =\frac{\sigma_{d}^{2}}{q} \sum_{i, j} g_{i j}^{2} \\
& =\frac{\sigma_{d}^{2}}{q} \operatorname{Tr}\left([G]^{\dagger}[G]\right),
\end{aligned}
$$

where $\operatorname{Tr}$ is the trace operator and $[G]^{\dagger}$ is the transpose of $[G]$.

It is easily shown that the random noise is minimised if a reconstruction array is used that is given by

$$
g_{i j}=\alpha_{i} m_{j i}-\beta_{i}
$$

where $\alpha_{i}$ and $\beta_{i}$ are dependent on the mask open fraction between the $i$-th source pixel and the detector. For cyclic configurations $\alpha_{i}=\alpha$ and $\beta_{i}=\beta$ for all $i$, hence reconstruction by this method is equivalent to the balanced cross-correlation technique proposed by Fenimore \& Cannon (1978).

Unfortunately, where the mask pattern does not have the perfect coding properties of URAs then for $[G]$ given by Eq. (6), although random noise is minimised, $[G][M] \neq$ $[I]$ and coding noise will be present in the reconstruction ${ }^{1}$. This will also be the case even for URA-based mask patterns if the recorded pattern is corrupted in some way, for example by obscuring support structure or by dead detector regions. In these circumstances it would be desirable to have a method of reconstructing an estimate of the source field with reduced coding noise whilst still suppressing random noise as much as possible.

Where it is applicable, Wiener filtering is just such a technique. Commonly associated with optical image processing (e.g. Andrews \& Hunt 1977), it is capable of compensating for the 'ill-conditioned' nature of a direct deconvolution function. In the Fourier domain the transform of the Wiener filter is defined by

$$
\mathcal{F}(W)=\frac{\mathcal{F}(M)^{*}}{|\mathcal{F}(M)|^{2}+r},
$$

where $\mathcal{F}$ denotes the Fourier transform operator, $r$ is the ratio of the noise power density to signal power density and ${ }^{*}$ denotes the complex conjugate. The application of Wiener filtering to coded aperture image reconstruction was first discussed by Sims et al. (1980). Unfortunately, use of the Wiener filter as defined in (7) requires that the system PSF is spatially (and temporally) invariant. In terms of coded mask instruments, this is only the case in the fully coded field of view of cyclic systems, where typically ideal mask patterns are used anyway and therefore the problems associated with coding noise do not arise. As a means of improving the reconstructed image quality for coded mask telescope configurations with imperfect coding properties Wiener filtering in the Fourier domain can at best be a rough approximation and has not found significant practical application.

We here propose a reconstruction method, based on the Wiener criterion using matrix formulation, which is a generalisation of Fourier Wiener filtering. It is applicable to all imaging systems where a coding matrix can be

\footnotetext{
${ }^{1}$ For URAs used in a cyclic configuration the matrix inverse and cross-correlation reconstruction techniques are equivalent, i.e. $[M]^{-1}=\alpha[M]^{\dagger}-\beta[J]$ where $[J]$ is filled with ones.
} 
formed, including those where the flux modulation has a time variable component.

\section{MMSE reconstruction}

According to the Wiener criterion, the estimate $[\hat{S}]$, of the original source field $[S]$ is constructed to minimise the mean-square-error, $\mathcal{E}^{2}$, given by

$$
\mathcal{E}^{2}=\operatorname{Tr}[E]([S]-[\hat{S}])\left([S]^{\dagger}-[\hat{S}]^{\dagger}\right) .
$$

Here, $[E]$ is a weighing matrix used to apply an independent weight to the solution of each source field pixel (the simplest form of $[E]$ is the identity matrix $[I]$ which applies equal errors to all points). Substituting $[\hat{S}]=[G][D]$ into Eq. (8) and minimising $\mathcal{E}^{2}$ with respect to $[G]$ gives (Helstrom 1967) a 'minimum mean-square-error' (MMSE) reconstruction matrix which is independent of the precise form of $[E]$,

$$
[G]=\left[\Phi_{\mathrm{s}}\right][M]^{\dagger}\left([M]\left[\Phi_{\mathrm{s}}\right][M]^{\dagger}+\left[\Phi_{\mathrm{n}}\right]\right)^{-1} .
$$

The matrices $\left[\Phi_{\mathrm{s}}\right]$ and $\left[\Phi_{\mathrm{n}}\right]$ are the source and noise covariance arrays respectively, and are given by

$$
\begin{aligned}
{\left[\Phi_{\mathrm{s}}\right] } & =\mathbb{E}[S][S]^{\dagger} \\
{\left[\Phi_{\mathrm{n}}\right] } & =\mathbb{E}[N][N]^{\dagger} .
\end{aligned}
$$

As the covariance matrices cannot be known exactly, it is in practise necessary to estimate their form prior to reconstruction. If the noise is 'white', then $\left[\Phi_{\mathrm{n}}\right]$ is diagonal and can be represented by $\left[\Phi_{\mathrm{n}}\right]=\phi_{\mathrm{n}}[I]$, where $\phi_{\mathrm{n}}$ is the variance of the detector data counts ${ }^{2}$. Similarly, taking a white source field distribution implies that Eq. (9) may be represented as

$$
[G]=[M]^{\dagger}\left([M][M]^{\dagger}+\Omega[I]\right)^{-1},
$$

or

$$
[G]=\left([M]^{\dagger}[M]+\Omega[I]\right)^{-1}[M]^{\dagger},
$$

where $\Omega=\phi_{\mathrm{n}} / \phi_{\mathrm{s}}$.

Unfortunately, if $[M]$ is of the form given in Eq. (1) the MMSE technique takes no account of the existence of detector background counts and a bias is introduced into the image. To overcome this we may redefine the coding equation to include 'pseudo-sources' from which we can assume the detector background intensities originate. Supposing that all detector elements are subject to a uniform background intensity $b$, we can rewrite the coding equation as

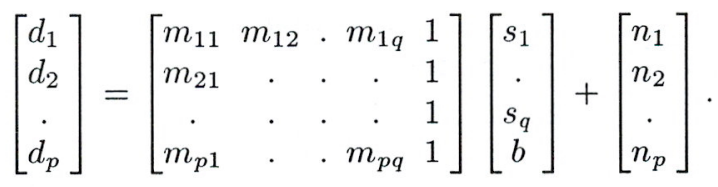

\footnotetext{
${ }^{2}$ It may be necessary to scale the detector data, making corresponding adjustments to the associated rows in the coding matrix, to make $\phi_{\mathrm{n}}$ the same for all elements.
}

It can be shown that in a low signal-to-noise ratio (SNR) environment, substituting a coding matrix of this form into Eq. (12) or Eq. (13) gives a reconstruction matrix of the form

$$
g_{i j}=\frac{1}{\Omega}\left(\alpha_{i} m_{j i}-\beta_{i}\right),
$$

where $\alpha_{i}$ and $\beta_{i}$ are equivalent to those of the crosscorrelation method in Eq. (6).

Although the reconstruction produced from $[G]$ given by Eq. (15) is identical in form and SNR to that created by a correlation method, the returned intensities are scaled down by a factor of $\Omega$. To obtain the absolute intensity of the source field distribution it is necessary to normalise $[G]$. The resulting form of the reconstruction matrix (referred to as $\left.\left[G^{\prime}\right]\right)$ is given by

$$
\left[G^{\prime}\right]=[\Psi]\left([M]^{\dagger}[M]+\Omega[I]\right)^{-1}[M]^{\dagger},
$$

where $[\Psi]$ is a diagonal matrix whose terms are designed to normalise $\left[G^{\prime}\right]$ such that $\sum_{j} g_{i j}^{\prime} m_{j i}=1$. Hence the diagonal terms of $[\Psi]$ are

$$
\psi_{i i}=\frac{1}{\sum_{j} g_{i j} m_{j i}} .
$$

In the limit where $\phi_{\mathrm{n}} \gg \phi_{\mathrm{s}}$ the normalised MMSE matrix $\left[G^{\prime}\right]$ gives a reconstruction identical to that of the correlation method.

Considering the opposite extreme, $\phi_{\mathrm{s}} \gg \phi_{\mathrm{n}}$, then Eq. (16) approximates to

$$
\left[G^{\prime}\right]=[G]=\left([M]^{\dagger}[M]\right)^{-1}[M]^{\dagger} .
$$

The matrix $\left([M]^{\dagger}[M]\right)^{-1}[M]^{\dagger}$ is equivalent to the MoorePenrose generalised inverse of $[M]$ (for review see BenIsrael \& Greville 1974). For the case where $[M]$ is overdetermined it follows that $\left[G^{\prime}\right][M]=[I]$. If $[M]$ is square and non-singular then in the high SNR environment, $\left[G^{\prime}\right]$ tends to the simple inverse $[M]^{-1}$.

The assumption of a white source field intensity distribution is justifiable where the field of view comprises a limited population of point source emitters, but it may not be appropriate in all cases. One such situation arises when the field contains so many point sources that the mean source pixel intensity, $\bar{s}$, is significantly greater than zero. A suitable source covariance matrix is then of the form

$$
\left[\Phi_{\mathrm{s}}\right]=\sigma_{\mathrm{f}}^{2}[I]+\bar{s}^{2}[J]
$$

where $\sigma_{\mathrm{f}}$ is the rms deviation in the source field intensity distribution about $\bar{s}$ and $[J]$ is a $q \times q$ matrix filled with ones.

Alternatively, there may be prior knowledge that the emission under study contains, or may contain regions of extended diffuse emission and such knowledge should be taken into account in constructing $\left[\Phi_{\mathrm{s}}\right]$.

Another case where an alternative form for $\left[\Phi_{s}\right]$ should be considered is when the chosen reconstructed image 
pixel size is less than the intrinsic instrument angular resolution (characterised, for example, by the angle subtended at the detector by a mask element). A strong correlation between some image pixels pairs will exist and for a high SNR $\left(\phi_{\mathrm{s}} \gg \phi_{\mathrm{n}}\right)$ using a white source field will lead to a PSF peak one image pixel wide, and hence potentially to considerable 'super-resolution'. This is obtained at the expense of a strong anti-correlation between close pairs of image pixels and exaggerated noise. In such cases a pragmatic approach is to use a source covariance matrix in which the $i j$-th element of $\left[\Phi_{\mathrm{s}}\right]$ is given by

$$
\phi_{\mathrm{s}, i j}=\phi_{\mathrm{s}, 0} \exp -\left(\frac{|i-j|^{2}}{2 \omega^{2}}\right),
$$

for example, where $\phi_{s, 0}$ is the expected covariance between pixels at zero lag and $|\boldsymbol{i}-\boldsymbol{j}|$ is the separation between the $i$-th and $j$-th source pixels. For fields of point sources $\omega$ may be set equal to the rms width of the nominal instrument PSF. It will generally still be reasonable to assume the noise is white. Incorporating this form of the source covariance matrix into the definition of the normalised MMSE reconstruction matrix implies that

$$
\left[G^{\prime}\right]=[\Psi]\left([M]^{\dagger}[M]+\phi_{\mathrm{n}}\left[\Phi_{\mathrm{s}}\right]^{-1}\right)^{-1}[M]^{\dagger} .
$$

If the coding matrix has been extended to contain transmission coefficients from one or more pseudo-sources it is important to include the covariance properties of the detector background in the definition of $\left[\Phi_{\mathrm{s}}\right]$.

\section{Simulated image reconstruction}

To compare the MMSE reconstruction technique, with the correlation and inverse methods, reconstructions of simulated data from the Galactic Centre have been made. The results of Goldwurm et al. (1994) were used as a basis for the simulations. The simulated data correspond to a static observation of the fully coded field of view through a random coded mask using a detector with perfect spatial resolution, subject to a uniform background intensity and Poisson statistics. The fact that the mask pattern is random and non-cyclic implies that the PSF is position dependent, hence Wiener filtering in the Fourier domain would not be appropriate.

Figure 1 shows reconstructed images from the simulated data and illustrates the improvement in image quality obtained using the MMSE reconstruction technique compared with that by cross-correlation. Reconstruction of the same data by the direct inverse method produces an image (not shown) so severely degraded by noise amplification that no sources are discernable. The statistics of the images are given in Table 1 . It is apparent from both Fig. 1 and Table 1 that in the intermediate SNR the MMSE reconstruction significantly reduces the coding noise compared with cross-correlation, improving the
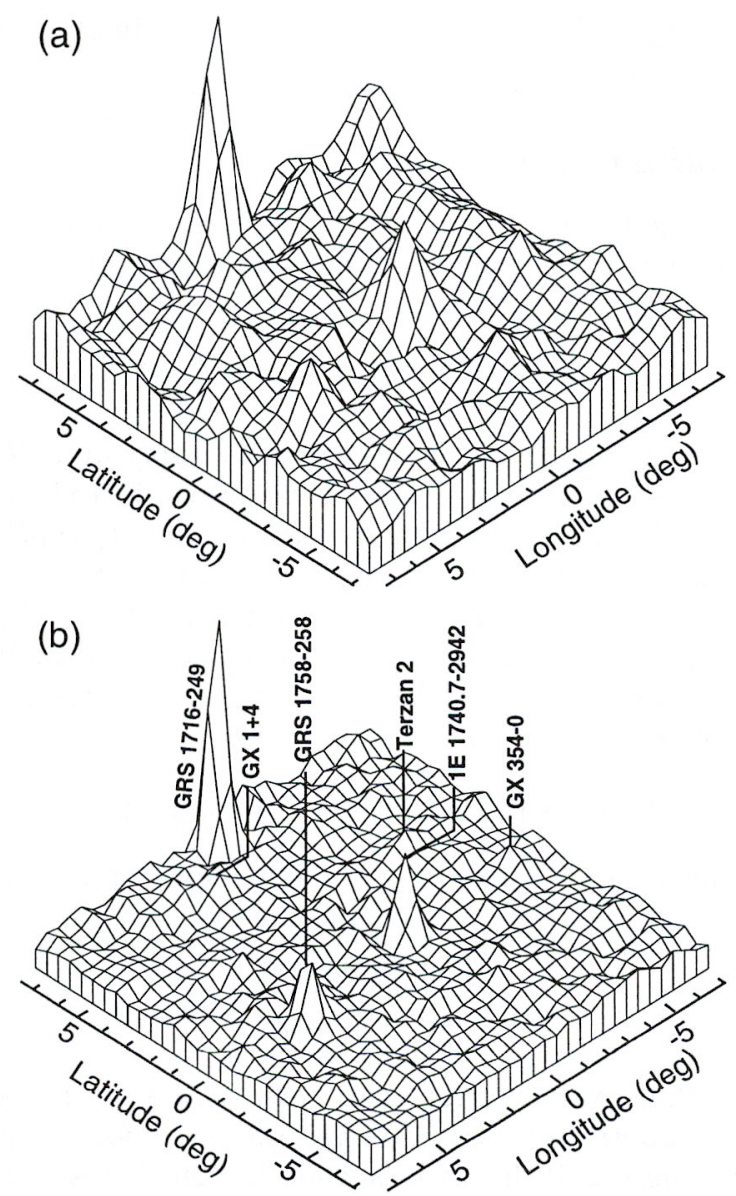

Fig. 1. Images reconstructed from simulated data using cross correlation a) and MMSE b) reconstruction techniques. The simulations assume a single observation of the Galactic Centre represented on a $30 \times 30$ grid of $0.5^{\circ}$ pitch through a large random coded mask pattern with $1^{\circ}$ elements. Data is assumed to be recorded with a $30 \times 30$ element detector plane subject to a uniform background and Poisson statistics. There are $8.310^{5}$ detected source photons and $8.510^{6}$ background events

'clarity' of the image and reducing the risk of source confusion. At the same time there is evidence of resolution enhancement.

The expected levels of random and coding noise in the reconstructed image can be calculated directly from the coding and decoding matrices, allowing a quantitative comparison of reconstruction techniques for a given system. Assuming that the coding noise $\left(\sigma_{\mathrm{c}}\right)$ and random noise $\left(\sigma_{\mathbf{r}}\right)$ are independent, the total level of fluctuations in the image will be given by

$$
\sigma_{\mathrm{t}}^{2}=\sigma_{\mathrm{c}}^{2}+\sigma_{\mathrm{r}}^{2}
$$

Figure 2 shows the reconstructed SNR for the previously described random system, plotted as a function of the simulated source SNR. The noise and source fields 
Table 1. Statistics of the simulated reconstructions shown in Figs. 1a-b. The areas covered by ghost sources at $>5 \sigma$ are quoted as a percentage of the source field area

\begin{tabular}{lccccc}
\hline Recon. & \multicolumn{2}{c}{ Sources } & \multicolumn{2}{c}{ Largest source } & Error \\
\cline { 2 - 5 } $\begin{array}{l}\text { method } \\
\text { (Fig. 1) }\end{array}$ & true & ghost & true & ghost & $\begin{array}{c}\text { rms } \\
(\sigma)\end{array}$ \\
\hline \hline (a) correl. & 6 & $>5 \sigma$ & $(\sigma)$ & $(\sigma)$ & $(\sigma \%$ \\
(b) MMSE & 6 & $2 \%$ & 66 & 8 & 123 \\
\hline
\end{tabular}

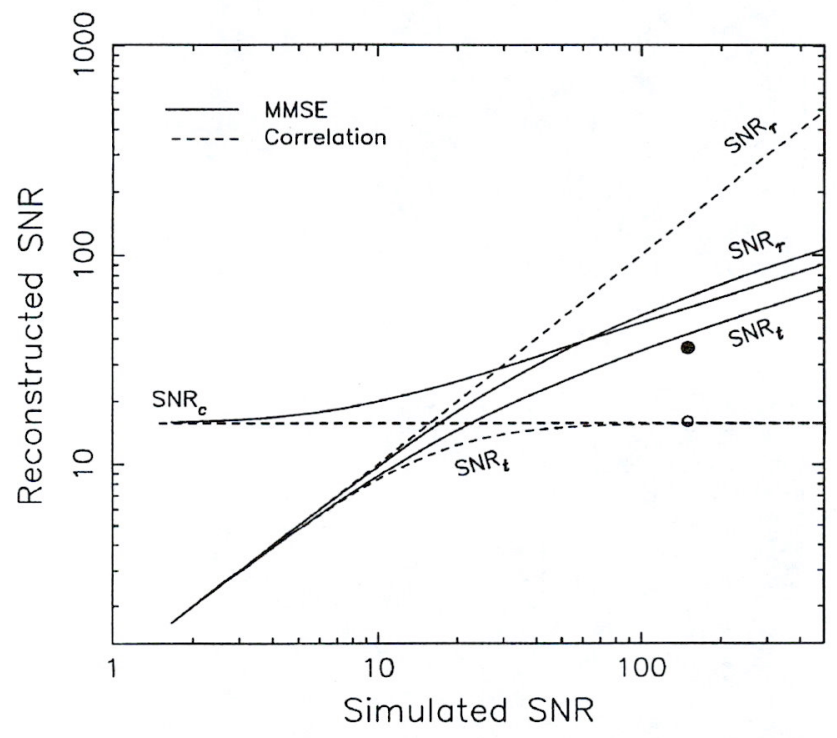

Fig. 2. The ratios $\left(\mathrm{SNR}_{\mathrm{c}}, \mathrm{SNR}_{\mathrm{r}}\right.$ and $\left.\mathrm{SNR}_{\mathrm{t}}\right)$ of the strength of a single point source to $\sigma_{\mathrm{c}}, \sigma_{\mathrm{r}}$ and $\sigma_{\mathrm{t}}$ using the MMSE (solid) and correlation (dashed) methods of image reconstruction. The hollow and filled circles represent the $S_{N R}$ with which the largest source is reconstructed in the images shown in Figs. 1a and $1 \mathrm{~b}$, respectively

are assumed to be white. Consistent with the results in Sect. 3, the performance of the MMSE reconstruction is seen to converge to that of the cross-correlation method at low SNRs where random noise dominates and crosscorrelation is optimum. If the SNR is high, so that coding noise is particularly important, then MMSE reconstruction tends to the inverse method. In intermediate SNR environments, the total noise is substantially lower than for the other linear reconstruction methods.

We note that the reconstruction technique proposed will only produce the MMSE image if the estimate of the SNR is correct, but we find that the accuracy with which the SNR must be determined is usually not great. Figure 3 illustrates that for the system described above, a range of assumed SNR values exists for which the MMSE technique increases the reconstructed SNR of a point source above that of a cross-correlation reconstruction. The range over

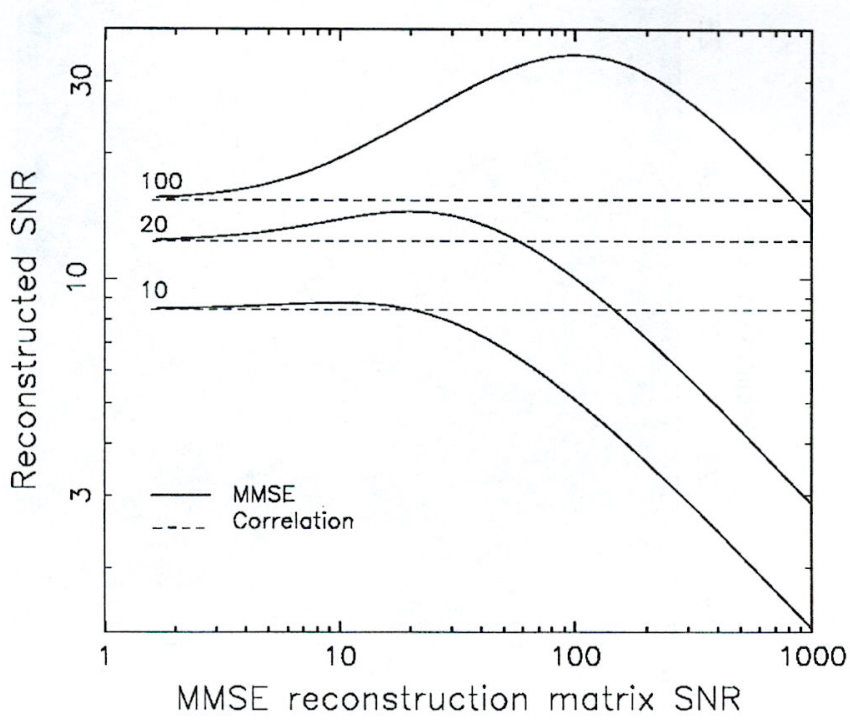

Fig. 3. The $\mathrm{SNR}_{\mathrm{t}}$ with which a single source pixel is reconstructed where the true $\mathrm{SNR}_{\mathrm{r}}$ is 100,20 and 10. Reconstruction uses a MMSE reconstruction matrix designed for various SNR environments

\section{Balloon flight data}

An experimental telescope designed to investigate the feasibility of using a compact array of germanium detector elements to produce hard X-ray images of the sky using coded aperture techniques was built at the University of Birmingham and flown on a high altitude balloon in 1993 (Skinner et al. 1995). It was carried as a 'piggy-back' experiment on board the GRIS payload (Tueller et al. 1994) and observed the X-ray emission from the Crab nebula and Cygnus X-1.

The instrument design was such that there was considerable source motion through the field of view, leading to temporal coding as well as spatial. In fact in the analysis it was necessary to the break the data from the $\sim 1$ hour long observations into discrete 30 second pointing phases.

Due to the random nature of the coded mask and the observing strategy used, the PSF of the experimental telescope was neither spatially nor temporally independent. In this case conventional Wiener filtering in the Fourier domain cannot be used as a means of producing reduced coding noise images. However, by implementing the MMSE image reconstruction technique it is possible to reduce the degrading effects of the coding noise and produce better quality images than those formed using the conventional cross correlation method.

Cross-correlation image reconstructions from the balloon flight data, are shown in Figs. $4 \mathrm{a}$ and $4 \mathrm{c}$ (these are essentially the same as those published by Skinner et al. 
(a)

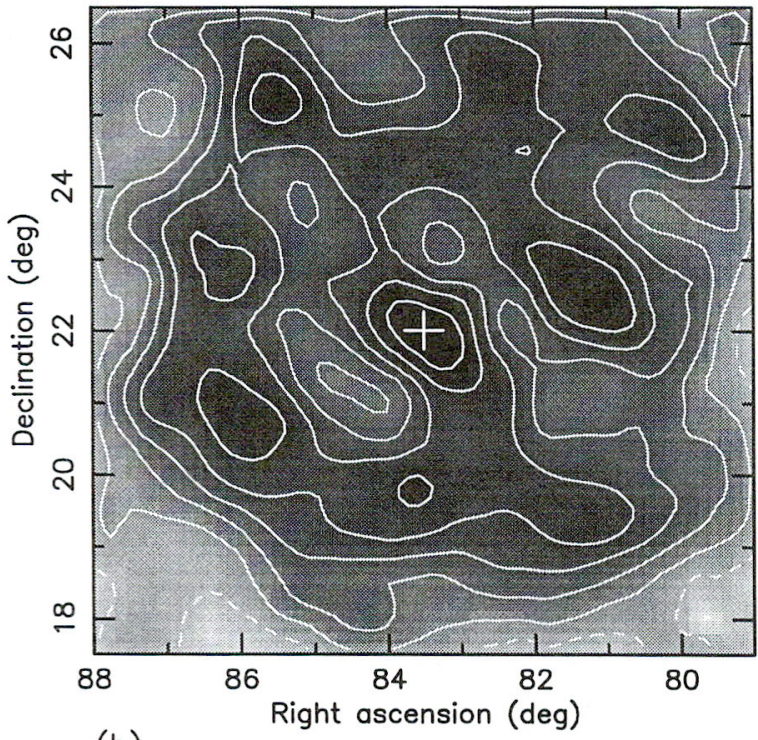

(b)

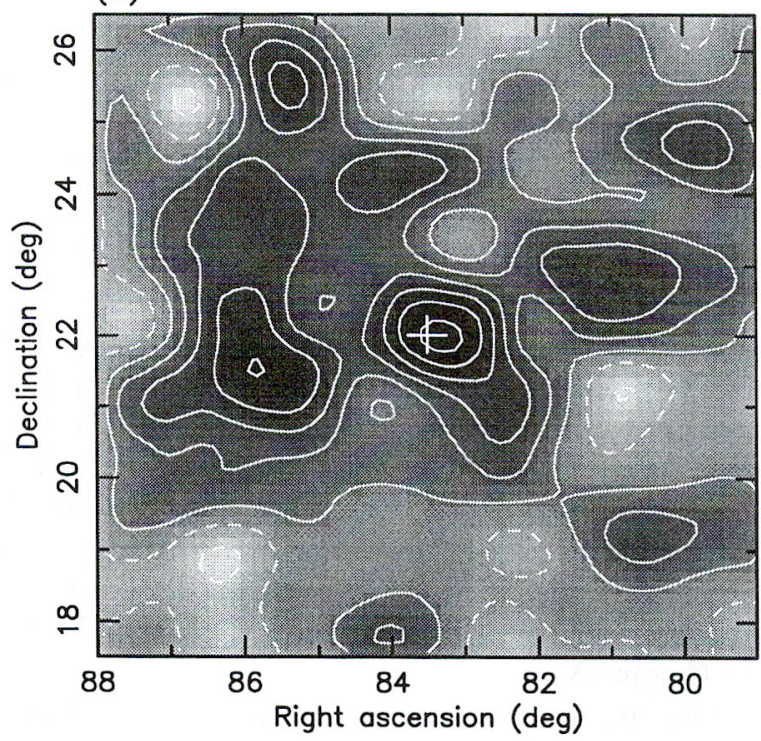

(c)

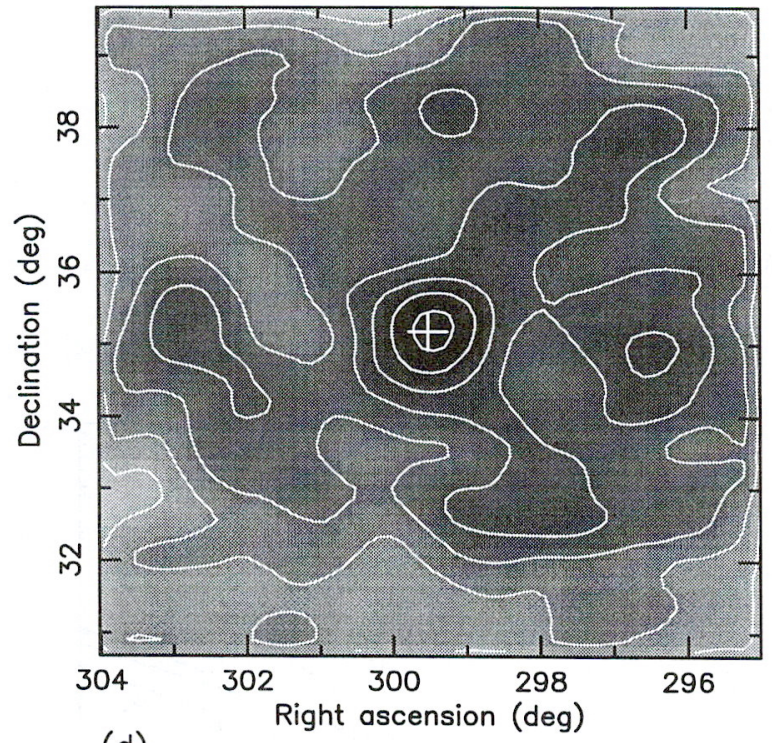

(d)

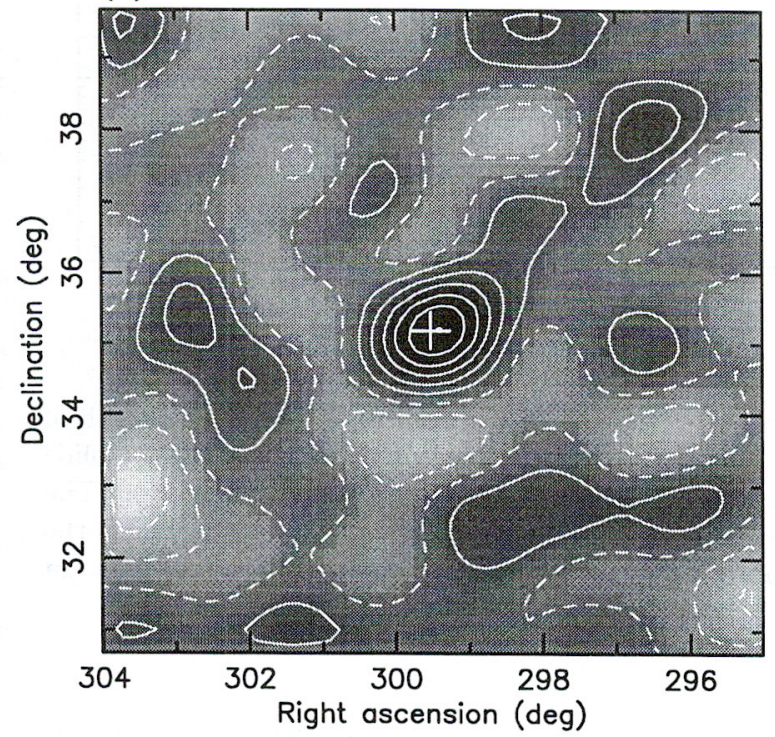

Fig. 4. Reconstructions of the Crab nebula a) and b) and Cygnus X-1 c) and d) observed between 30 and $80 \mathrm{keV}$ using cross-correlation a) and c) and MMSE b) and d) reconstruction techniques. Images are significance plots with grey-scales adjusted to correspond with the full range of values in each plot. Contours are at $1 \sigma_{\mathrm{r}}$ intervals with dashed contours representing $\leq 0$ significance. In each case the images were formed by solving a $30 \times 30$ element source field with $0.3^{\circ}$ resolution. The reconstructions were then $\times 2$ over-sampled along both axes and smoothed with a normalised Gaussian $\left(\mathrm{FWHM}=0.3^{\circ}\right)$. The crosses indicate expected source positions. Coordinates are J2000

$\left.1995^{3}\right)$. Reconstructions of simulated data showed that

\footnotetext{
${ }^{3}$ The original cross-correlation images presented in Skinner et al. (1995) were obtained assuming a constant variance $\left(\bar{\sigma}_{d_{k}}^{2}\right)$ for each detector $k$ throughout the observation and gave rise to a slightly lower peak significance in the reconstructed image of Cygnus X-1 than those shown here.
}

the intensity fluctuation surrounding each source position is consistent with the expected coding noise due to the target source emission (Skinner et al. 1995).

Figures $4 \mathrm{~b}$ and $4 \mathrm{~d}$ are corresponding images of the Crab nebula and Cygnus X-1 reconstructed using the MMSE matrix given by Eq. (21). It is apparent that the magnitude of the coding noise has been reduced and the 
images contain less ambiguity than exists in the crosscorrelation reconstructions. All the images presented are 'significance plots' in which the intensity in each pixel is shown relative to the noise level predicted for that pixel from random noise alone. Thus the rms level away from the source peaks would be 1 if only random noise were present. Ignoring the central peak, the rms noise in the cross-correlation images of the Crab nebula and Cygnus $\mathrm{X}-1$ are in fact 2.99 and 3.75 respectively. Using Eq. (22), this implies that the coding noise exceeds the random noise by factors of 2.82 and 3.61. Evaluating the fluctuations in Figs. $4 \mathrm{~b}$ and $4 \mathrm{~d}$ in the same way indicates that the MMSE reconstructions contain total noise reduced to 1.75 and 0.95 times the random noise, demonstrating a significant improvement over the cross-correlation technique. These figures are closer to the value $(\sqrt{2})$ expected for a system which balances the magnitudes of the coding and random noise.

\section{Conclusions}

The matrix formulation of MMSE reconstruction method presented above has been shown to balance the effects of random detector noise and imperfect coding to give better quality images than those obtained by the correlation or matrix inverse methods. In a high SNR environment the MMSE reconstruction tends to that of the matrix inverse, and where the SNR is low it gives a result equivalent to the correlation technique. It can be shown (Andrews \& Hunt 1977) that in cases where the coding matrix is block circulant and the source and noise covariance matrices are stationary, separable and independent, the MMSE reconstruction matrix can be simplified to the Fourier definition of the Wiener filter given by Eq. (7). The MMSE reconstruction matrix is therefore a generalisation of the Fourier Wiener filter and is applicable to a much wider variety of instrument configurations. The method is most effective in the intermediate SNR domains and when applied to systems with imperfect coding properties perhaps arising from dithered observations of the source field, as is currently being considered for the INTEGRAL mission.

Relatively recently, the method of optimising image quality by using the MMSE reconstruction technique would have been impractical. The inversion of large matrices is very computationally demanding and the reconstruction of usefully large source fields with an acceptable resolution would have taken an unreasonable amount of time. Currently, the practical size of the task which can be undertaken using a 'fast' computer to perform MMSE reconstruction is still limited to a certain extent by the problems of storing and manipulating large matrices. However we have demonstrated that the technique is practicable for realistic problems and improvements in computer technology will extend its range of applicability further.

Acknowledgements. We are grateful to P. Connell and I. Stevens for useful comments and advice. This work has made extensive use of the Starlink network.

\section{References}

Andrews H.C., Hunt B.R., 1977, Digital Image Restoration, Prentice-Hall, London

Ben-Israel A., Greville T.N.E., 1974, Generalised Inverses Theory and Applications, Wiley-Interscience, New York

Caroli E., Butler R.C., Di Cocco G., Maggioli P.P., Natalucci L., Spizzichino A., 1984, Il Nuovo Cimento 7, 786

Caroli E., Stephen J.B., Di Cocco G., Natalucci L., Spizzichino A., 1987, Space Sci. Rev. 45, 349

Dicke R.H., 1968, ApJ 153, L101

Fenimore E.E., 1978, Appl. Opt. 17, 3562

Fenimore E.E., Cannon T.M., 1978, Appl. Opt. 17, 337

Fenimore E.E., 1980, Appl. Opt. 19, 2465

Goldwurm A., Cordier B., Paul J., et al., 1994, Nat 371, 589

Gottesman S.R., Fenimore E.E., 1989, Appl. Opt. 28, 4344

Gourlay A.R., Stephen J.B., 1983, Appl. Opt. 22, 4042

Gunson J., Polychronopulos B., 1976, MNRAS 177, 485

Helstrom C.W., 1967, J. Opt. Soc. Am. 57, 297

Rideout R.M., 1996, Coded imaging systems for X-ray Astronomy, Ph.D. Thesis, University of Birmingham

Sembay S., Gehrels N., 1990, Nucl. Instrum. Meth. 295, 477

Sims M.R., Turner M.J.L., Willingale R., 1980, Space Sci. Instrum. 5, 109

Skinner G.K., Balthazor R.L., Herring J.R.H., et al., 1995, Nucl. Instrum. Meth. 357, 580

Skinner G.K., Ponman T.J., 1995, Inv. Prob. 10, 655

Tueller J., Barthelmy S.D., Bartlett L.M., et al., 1994, (submitted to ApJ) 